\title{
Crystalline Lens Imaging with a slit-scanning system
}

Cristina Oliveira, José B. Almeida, Sandra Franco ${ }^{a}$

\section{Introduction}

The human crystalline lens plays a fundamental role on the retinal image quality. The ability of the lens to change its shape and, consequently, the total refractive power of the eye, during accommodation, enables one to focus objects at a wide range of distances. Knowledge regarding the crystalline lens' geometric properties provides insights into the focusing mechanism of the visual system. Namely, the study of changes in surface shape and curvatures of the lens during accommodation is essential for a better understanding of the accommodation mechanism itself $[1,2]$; the age-related changes in these geometric properties of the lens are necessary to explain the etiology and progression of presbyopia $[3,4]$. Furthermore, crystalline lens' optical properties, such as focal length and spherical aberrations, are closely related to its geometric properties.

The aim of this work is to present a slit-scanning tomography system that's capable of imaging the anterior chamber of the eye. We overview the applicability of our device to the crystalline lens.

\section{Optical System Configuration}

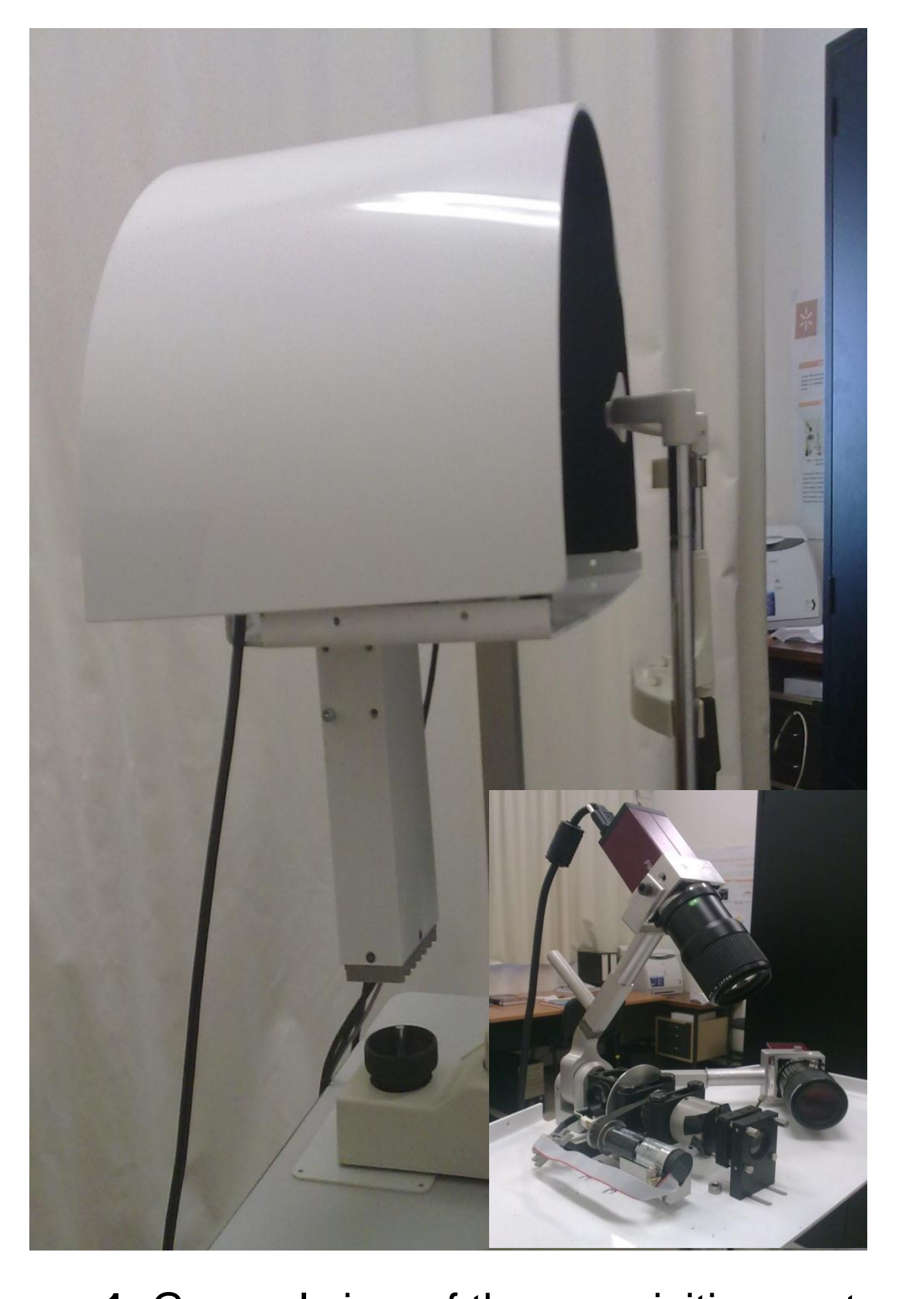

The tomography system (figure 1) is based on the slit-scanning principle and is essentially composed by two components: a compact illumination system, that expands the light beam in a fan, and an observation system that captures the images for posterior analyses.

Figure 1. General view of the acquisition system.

\section{ILLUMINATION SYSTEM}

The illumination system is composed by a monochromatic blue light-emitting diode (LED), a collimator, a cylindrical lens, a convex lens and an apodizing aperture slit. After the beam is collimated, the cylindrical lens expands it into a fan. This cylindrical lens is attached to a rotational motion system controlled by the customized software that allows to explore the entire cornea. The fan is focused on the cornea surface by the convex lens and the light diffused produces an optical section.

\section{OBSERVATION SYSTEM}

The observation system integrates two Charge Coupled Device (CCD) cameras that captured the optical sections of the cornea. These two cameras act like a single virtual camera that rotate with the cylinder lens.

\section{Image Acquisition and Analysis}

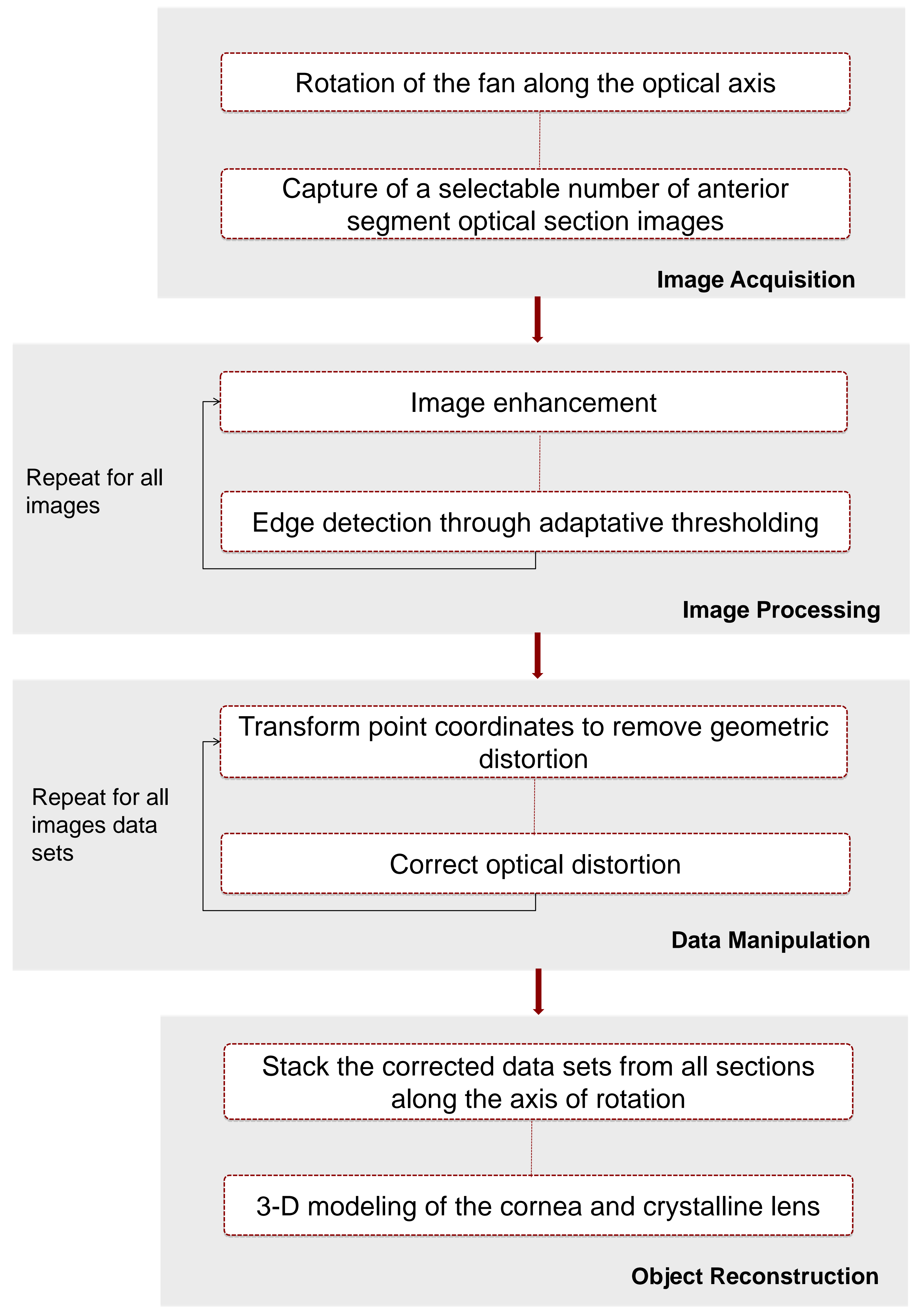

\section{Results}

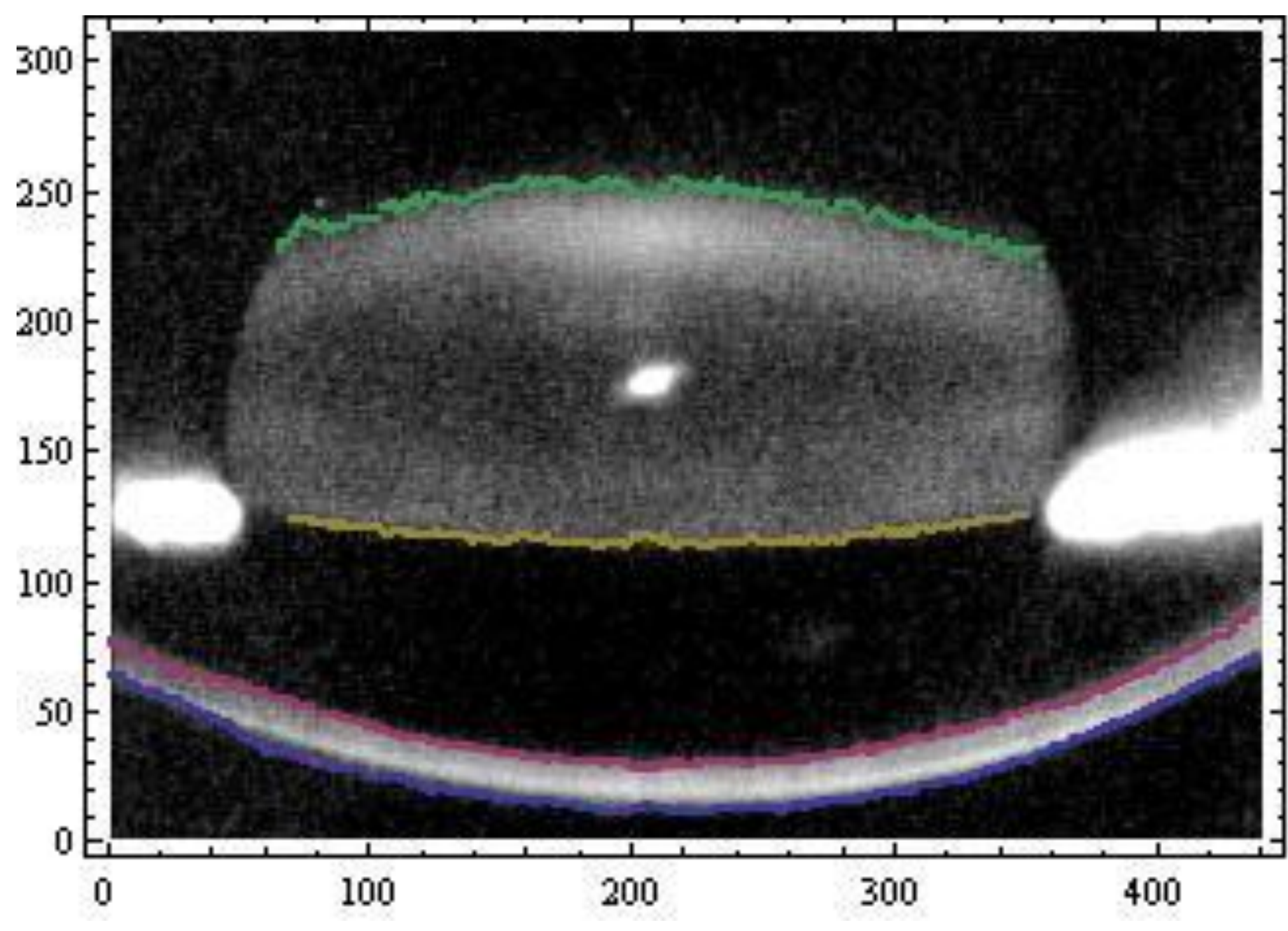

$\left\{\begin{array}{c}\text { Repeated for the six } \\ \text { acquired optical }\end{array}\right.$ acquired optical
sections

Figure 2. Anterior segment optical section after both corneal and crystalline lens surfaces detection.

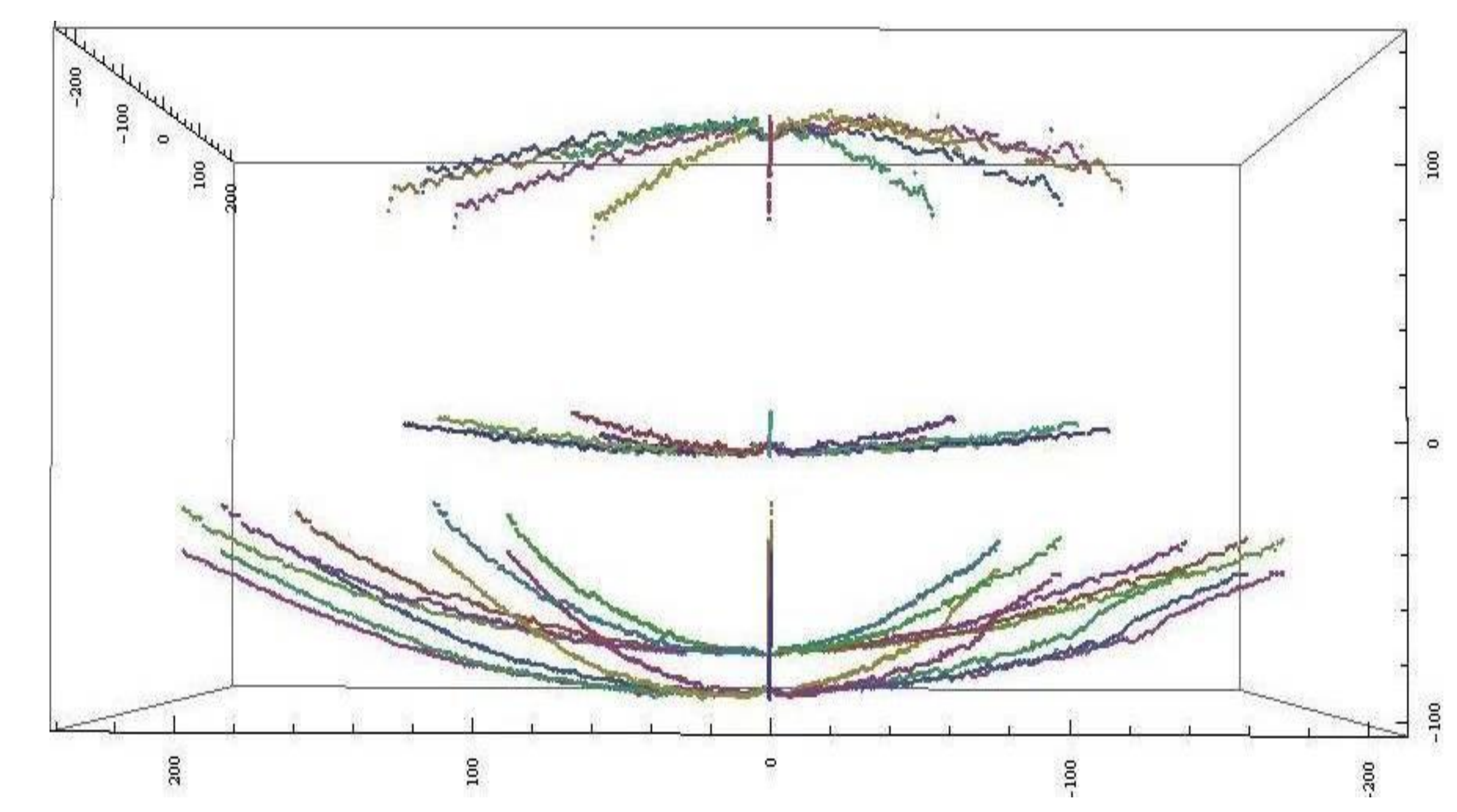

Figure 3. Profile of anterior and posterior surfaces of the cornea and of the crystalline lens (from bottom to top).

\section{Conclusions \& Future Work}

We described a slit-scanning tomography system and its application to imaging the anterior segment structures, particularly the crystalline lens. The developed algorithm, based on gray-scale analyses, provided an effective detection of both anterior and posterior surfaces of the crystalline lens. Therefore, we were able to reconstruct the 3-dimensional structure of the lens.

This system has already been successfully used to perform complete threedimensional quantitative analyses of the cornea, including:

CORNEAL TOPOGRAPHY
CORNEAL PACHYMETRY
CORNEAL ABERRATIONS

The resultant 3-D model of the crystalline lens can be used to derive several geometric parameters, such as surfaces' shape and curvature, as well as its thickness.

\section{References}

1.Chien, Chang-Hai M, Tseng Huang, and Ronald A Schachar. 2006. Analysis of human crystalline lens accommodation. Journal of biomechanics 39, no. 4:672-80.

2.Dubbelman, $M, G L$ Van Der Heijde, and $H$ A Weeber. 2005. Change in shape of the aging human crystalline len with accommodation. Vision research 45, no. 1: 117-32.

3. Koretz, J F, C A Cook, and P L Kaufman. 1997. Accommodation and presbyopia in the human eye. Changes in the anterior segment and crystalline lens with focus. Investigative ophthalmology \& visual science 38, no. 3: 569-78. 4. Weeber, Henk a, and Rob $G L$ van Der Heijde. 2007. On the relationship between lens stiffness and accommodative amplitude. Experimental eye research 85, no. 5.6027 